\title{
REPAROS DE JUAN IRIARTE AL DICCIONARIO DE AUTORIDADES
}

\author{
CARMEN HERNANDEZ GONZALEZ \\ (Universidad de Valladolid)
}

La figura de Juan Iriarte es todavía hoy una de las sombras de nuestro siglo de las luces. Mucho desconocemos de sus estudios y aportaciones en particular a la Real Academia Española de la Lengua, institución de la que fue miembro de número, tesorero y secretario interino.

Este humanista nació en el Puerto de la Cruz. Se educó en París, siendo condiscípulo de Voltaire. Lo encontramos en Madrid en 1724, lugar en el que se desarrollaría toda su existencia, como preceptor, primero, y más tarde, en diversos cargos dentro de la Biblioteca Real: en 1729 oficial escribiente y en 1732 bibliotecario de asiento. Fue también traductor de la primera secretaría de estado, gracias a sus amplísimos conocimientos de latín, que le Ilevaron a elaborar durante toda su vida una Gramática latina (Iriarte, 1771), que no llegaría a ver impresa, y multitud de epigramas -en sus Obras sueltas (1774) y varios manuscritos existentes en la Biblioteca de B. March: Opúsculos, epigramas y poesías sueltas (1798), Epigramas y sátiras. Versión latina. Epigrammatum delectus a Ionne Iriarte concinnatum. Anno 1773; vid. noticia de los manuscritos en Aguilar Piñal (1986)-, género para el que estaba muy bien dotado (vid. para este último aserto y para la biografía a Cotarelo y Mori, 1897).

Supernumerario en la Academia Española desde 1743, ocupó el sillón Z de la docta casa en 1747, y también fue socio de honor de la Academia de Bellas Artes de San Fernando desde 1752. Sus dotes de bibliógrafo, latinista, filólogo, crítico y erudito quedaron patentes para sus contemporáneos por sus publicaciones en el Diario de los literatos a partir de 1737, alguno de los cuales están reeditados en las Obras sueltas (1774), y por sus Regiae Bibliothecae Matritensis Codices Graeci Mss. Joannes Iriarte... (1769) y, la anteriormente citada, Gramática latina (1771) que alcanzó nueve ediciones en España y varias en Francia e Inglaterra (Palau, 1954; Aguilar Piñal, 1986). Iriarte murió en Madrid en 1771.

Biografías y estudios sobre este escritor han sido realizados, en primer lugar, por Bernanrdo Iriarte (1771) -hermano del famoso fabulista Tomás- y, luego, levemente modificado en la introducción a las Obras sueltas (Iriarte, 1774) de áquel. Se han ocupado también de este autor Viera y Clavijo (1777-83), Sempere y Guarinos (1785-89), Cotarelo y Mori (1897), Menéndez Pelayo (1974), J. M de Cossío (1926), Guigou y Costa (1945), Millares Carlo (1981) y Aguilar Piñal (1986).

La importancia del mayor de los Iriarte ha sido menospreciada, a pesar de juicios 
como éste de Lázaro Carreter (1985, p. 195): "La Gramática de la Academia es muy superior, como compendio y ordenación de doctrinas anteriores, y es este el valor que posee en la historia gramatical española. En su elaboración habían intervenido, a lo largo de medio siglo, numerosos académicos, entre los que destacaron Luzán y el docto humanista don Juan de Iriarte, que contribuyó además a su elaboración, con una serie de comunicaciones, recogidas en el segundo tomo de las Obras sueltas (1774)". Efectivamente, en este segundo tomo podemos encontrar trabajos como "Advertencia preliminar para la Sintaxis Castellana" (Discurso I), "Observaciones sobre el adverbio" (Discurso VII), etc. Tiene también Juan Iriarte obras manuscritas como la Ortografía española, Partes de la oración, Diccionario de voces nuevamente introducidas en la lengua castellana, Concordancias de sustantivos y adjetivos o el texto que hoy editamos, Reparos / Sobre los primeros Pliegos / del Diccionario / corregido, notas inéditas que adjuntó junto con dos cartas a D. Francisco Antonio de Angulo, secretario de la Academia en estos años. Este texto, y otros que daremos a la imprenta en trabajos sucesivos, a la par que muestra parte de su labor en los trabajos cadémicos nos configura el perfil de este filólogo que debemos recuperar.

El autógrafo, pues tal lo creemos, se encuentra en la Biblioteca de B. March dentro del fondo denominado "J. Iriarte". Este manuscrito está, en compañía de otros varios, en el primero de los dos legajos que componen el volumen titulado Papeles de la Real Academia, y que tiene por signatura 14-2-2 I. El texto que aquí ofrecemos ocupa los folios 349-361 (mantenemos la numeración original).

Los criterios de edición que seguimos son los habituales: normalización de la puntuación y acentuación, mantenemos las grafías (así las mayúsculas) y las abreviaturas, subrayamos las palabras que no lo están según los criterios actuales y se separan las palabras que pudieran ir unidas.

\section{Reparos / Sobre los primeros Pliegos / del Diccionario / corregido}

Fol. 1 Los más graves autores dan por cosa sentada que el Aleph de la lengua Hebrea y el Aliph o Eliph de la arábiga, a que según el dictamen de la Academia, corresponde la $A$ del Abecedario Castellano, no son letras sino espíritus suaves.

Para evitar objeción tan poderosa, como también para dar a este artículo la más clara y precisa explicación, pudiera éste ceñirse a los criterios subsiguientes:

$" A$, primera Letra del Alafabeto, o Abecedario, de nuestra Lengua, y primera de nuestras Vocales. Hemos tomado de la latina su carácter, o figura mayúscula, y la usamos principalmente en los escritos impresos. Su nombre suena lo mismo que su potestad o valor; y su pronunciación es tan fácil y natural que se executa con abrir la boca y arrojar el aliento, pero apartando más el labio inferior del superior que en la pronunciación de las demás vocales; de la manera que la figura de la boca se manifieste algo prolongada (f. 349)".

Fol. 1b A la explicación aquí puesta puede substituirse la siguiente. "A como Parte de la Oración tiene en Castellano infinitos usos. Ya se usa como Verbo, ya como Preposición o Partícula, o ya como Interjección en la forma que se sigue".

Póngase luego en Artículo aparte las palabras: $A$ era etc. hasta $A$ hora, que se excluirá.

Fol. $4 \mathrm{~A}$ en las Universidades. El uso de las Universidades no me parece del caso para probar que la $A$ sea abreviatura Castellana, porque siendo la Lengua Latina la Lengua propia de ella , es más regular creer que la $A$ sea abreviatura del latín Approbatus que del 
castellano Aprobado, como la $\mathrm{R}$ de Reprobatus, que no de Reprobado. A cuya razón se puede añadir que las Universidades imitaron en esto la costumbre de los antiguos Tribunales Romanos, que usaron la $A$ y la $C$ como Abreviaturas de los verbos $A b s o l v o$ y Condenno. Y así, en lugar del referido exemplo de la Abreviatura de la A, juzgo sería más conveniente poner el de la $\mathrm{A}$ que quando está precedida la $\mathrm{V}$. con punto significa Alteza.

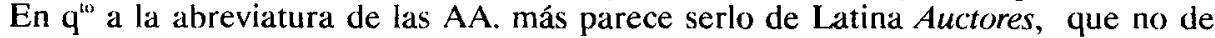
la castellana Autores; pues de aquella se ha usado mucho y se usa en los Escritores Latinos, de donde se les ha pegado la costumbre a algunos facultativos escribiendo en nuestra lengua. Y así no se debe reputar por Abreviatura Casetallana, al modo que no se debe tener por tal Abreviatura v.g., sino por tan Latina como las mismas voces que denota y de que ha sido señal antiquíssima en las obras escritas en este Idioma. Todo lo qual persuade a que se excluya de nuestro diccionario la expresión de tal Abreviatura.

Fol. 6 Abada etc. Procurando defender el P. Bluteau ' en su Diccionario Portugués ser falsa la sentencia de los que tienen a la Abada y al Rinoceronte por un mismo animal, debiera la Academia Española para sostener la verdad de estar en su Diccionario, afianzarse en razones más poderosas que el argumento de que se vale aquel Escritor, fundado en la descripción circunstanciada que (f. 351) trahe de la Ahada. Esta la ha tomado fielmente (como lo he averiguado) de la que trahe Dappers ${ }^{2}$ en su Descripción de Africa; y de su contexto resulta ser la Abada una fiera enteram ${ }^{16}$ diversa del Rinoceronte. Contra un argumento tan sustancial iqué fuerza pueden tener conjeturas tomadas ya del nombre de aquélla, ya del dibujo de éste formado por Juan de Arfe ${ }^{3}$.

En quanto a las primeras, no es tan cierto, como se supone, que el nombre de $A b a d a$ o Bada que parece el primitivo (Al pie. Nota."Es mui verosímil que el nombre Bada sea el primitivo, Indiano o Africano, y que habiéndole añadido los Portugueses el artículo $A$ de su lengua, que equivale al nuestro Castellano $L a$, y que de aquel nombre y este artículo se formase después la voz $A b a d a$, y dixesen separadamente La Bada. Lo cierto es que de la voz sencilla Bada usan algunos Escritores Portugueses como lo afirma Bluteau), sea originalm" Portugues; pues Covarrubias ${ }^{4}$ asegura que ser Indiano y yo le creyera Africano, respecto criarse este animal en varias partes de Africa, siendo mucho más creíble que una nación extrangera, al ver una fiera que ni ha visto ni conocido, se conforme en llamarla como la llaman los naturales del País donde se cría, que no el que la aplique un nombre tomado de su propio idioma. Así lo vemos paracticado por nuestros Españoles en las Indias Occidentales, de cuyas naciones han usado las voces para significar varias especies de Animales y otras cosas pertenecientes a aquel Nuevo Mundo. Además de esto, es mui verosímil que los Portugueses se valiesen del nombre Bada Africano para llamar la

'Se trata de Raphael Blutcau, autor de un Vocabulario portuguez e latino (1712-1721).

${ }^{2}$ Tiene que ser Oliverio Drapper, médico y geógrafo holándes muerto en Amsterdam en 1690. Su obra completa se publicó con el título Dapperus exotus curiosus y tiene varias obras sobre Africa: Nueva descripción de los países africanos (1668), Nueva descripción de las islas de Africa (1676) y Nueva descripción de Africa $(1680)$.

${ }^{3}$ Juan Arfe (nacido en León en 1535) fue platero y grabador. Hizo trabajos por encargo de Felipe II y escribió varios libros sobre su especialidad.

4 Vid. S. Covarrubias (1977). 
expresada fiera, al modo que tomaron el de Ganda Indiano para denotar al Rinoceronte, como lo asegura el mismo Bluteau con autoridad de Barros s. Con lo qual se puede confirmar la sentencia de ser estos animales distintos, pues les aplican distintos nombres y, por consiguiente, que $A b a d a$ o Bada no es lo mismo que Rinoceronte.

Por lo que mira a las segundas conjeturas fundadas en las palabras de Juan de Arfe, éstas pudieran hacer fuerza si dixese que dibuxaba a la Abada. Pero lo que dice este Autor es que dibuxa al Rinoceronte (f. 353), y su dibuxo corresponde realmente con la estampa, o lámina que de este animal han publicado los escritortes más acreditados, y con la que actualmente corre sacada del mismo que se ha visto en nuestros días en Nápoles y otras partes. Tampoco vale la conjetura de que la fiera que vio Arfe sería la misma Abada que se vio en Madrid en tiempo de Phelipe $2^{\circ}$, pudiendo haber sido alguno de los Rinocerontes que, según refiere Porreño ${ }^{\circ}$, mandó traher entre otras fieras extrañas aquel Monarca, también pudo haberle ${ }^{7}$ visto fuera de Madrid, o de España. Y aquí se ofrece naturalmente la reflexión de que si la Fiera que vio, describió y dibuxó un escritor y Artífice tan exacto como Arfe hibiese sido la Abada que se veía en aquel tiempo en Madrid, no hubiera dexado de darle este mismo nombre con que allí era conocida, para proceder en todo con mayor distinción y exactitud.

A estas razones y pruebas se reducen las que alegan la Academia a favor de su sentencia. Pero como quiera que éstas no parezcan suficientes para resolver la question de si la Abada y el Rinoceronte son un mismo animal, se hace preciso, en caso de que no se dé a Dappers el debido crédito, buscar otro Autor más instruido y más acreditado que determine enteram ${ }^{\text {te }}$ la dificultad. Y si esto no pudiere conseguirse, conténtese la Academia con suspender su juicio, apuntando brevemente en este Artículo la variedad de opiniones que hai sobre este punto, pues, me parece, saldrá más airosa con la duda que con la decisión (f. 355) ${ }^{3}$.

Fol. 10 Abalgar. Reconózcase la autoridad para saber qué especie particular farmaceútica sea esta.

Fol. 14 b Averígüese lo que es propiamente Abarcar el viento, para dar a esta expresión la equivalencia latina; y, asimismo, véase si las dos acepciones que están contiguas se

\footnotetext{
${ }^{5}$ Tiene que ser Juan de Barros, historiador portugués nacido en Vizeu en 1496 y muerto en 1570 . Escribió por encargo de Juan III una Historia Cieneral de la India, a la que parece referirse Iriarte.

${ }^{6}$ Baltasar Porreño nació en Cuenca a linales del siglo XVII. Escritor y sacerdote, ligura cn el calálogo del Diccionario de Autoridades. La obra de la que toma el dato Iriarte es Dichos y hechos del señor rey don Phelipe "el Pnidente", potentísimo y glorioso monarca de la España y de las Indias, Sevilla, 1639. (Hay una $3^{\text {a ed. en }}$ 1748).
}

\footnotetext{
${ }^{7}$ Leísmo del autor.
}

"Corominas y Pascual (1980-1983) definen abada: "rinoceronte", del port. abada y éste del malayo bādg íd. $1^{\text {a }}$ doc.: 1582. / En portugués bada desde 1541 hasta el siglo XVIII; la variante moderna abada; debida a la aglutinación del arlículo portugués, no se halla allí hasta 1611; en castellano enconl ramos también bada en varios autores del fin del s. XVI y princ. del s. XVII. Por error afirma Aut. que sólo designa la hembra del rinoceronte". 
Fol. 16 b. Abarrotar ${ }^{9}$. Explíquese qué cosa es barrote en la Naútica, si es instrum ${ }^{\text {to }}$ de hierro o de madera, para aplicar a este verbo la correspondencia Latina. Examínese, asimismo, si acaso se han multiplicado las significaciones del verbo Abarrotar.

Fol. 18. Abastecer. Parece que la significación de este verbo se debe extender a la de proveer no sólo de bastim ${ }^{\text {tos }}$, sino también de otra qualquiera cosa necesaria, como se infiere de los textos con que se autoriza este mismo verbo en ntr. Diccionario impreso. Y en caso de admitir esta extensión, será preciso mudar la correspondencia Latina que se le ha puesto.

Madrid y Mayo 20 de 1766

J. Y. (f. 357)

Papel que, acompañando a estos Reparos, remití a $\mathrm{D}^{\mathrm{n}}$ Francisco de Angulo ${ }^{10}$, Secretario de la Academia Española.

$A m^{\prime \prime}$ y $\mathrm{S}^{\mathrm{r}}$ :

Paso a mano de Vm. esos Reparos sobre los primeros pliegos del Diccionario corregido. No me ha sido posible remitirlos antes, habiéndose atravesado la corrección de las pruebas de la Bibliotheca Griega que está a mi cargo.

Sup $^{\text {to }}$ a Vm. se sirva hacer pres ${ }^{\text {te }}$ a la Academia esta escusa de mi tardanza; y mandar como spre. $q^{\text {(") }}$ sea de su agrado a su más afecto amigo y seg ${ }^{\circ}$ serv ${ }^{r}$

Hoy 20 de Mayo de 1766

$$
\text { J. Y. }
$$

\section{Amigo y $S^{\text {or: }}$}

Devuelvo a V. M. los diez primeros pliegos mstos. del I Tomo del Diccionario, en los quales van puestas las correspondencias Latinas " en la mejor forma que me ha sido posible. Siento infinito la tardanza ocasionada de mis frequentes impresiones (?).

No remito adjunto los Reparos y que tengo hechas sobre los referidos pliegos, por no estar todavía trasladados en limpio. Luego que lo están, que será mui pronto, procuraré remitir a $V^{a} \mathrm{M}$, Repitiéndome entre tanto a su disposición con las mismas veras con que ruego a $\mathrm{N}^{\mathrm{ru}}$. $\mathrm{S}^{\mathrm{r}}$ le $\mathrm{g}^{\mathrm{de}}$

${ }^{9}$ Según Timoteo O’Scanlan (1831), Abarrotar es "Nav. y Man. Apretar, asegurar la estiva, llcnando sus huecos con efectos a propósito || Cargar un buque aprovechando hasta los sitios más pequeños de su bodega y cámaras. En una y otra accpción sucle también decirse atorar y embarrotar".

${ }^{10}$ Francisco Antonio de Angulo cra el secretario de la Academia y uno de los miembros de la misma que más se dedicó a los trabajos de la ilustre corporación. Como secretario firma la Gramática de 1771.

" Como especialista en latín, era lógico que éste fuera uno de sus principales cometidos. Las correspondencias latinas, que tanto obsesionaron al bueno de Iriarte, fucron climinadas del Diccionario en la $11^{\text {a }} \mathrm{ed}$. , en 1869. 
$S^{(x)} D^{n}$ Fran $^{\infty}$ de Angulo

\section{Referencias bibliograficas}

Aguilar Piñal, F. (1986): Bibliografia de autores españoles del siglo XVIII, IV, Madrid, CSIC. Bluteau, R. (1712-1721): Vocabulario portuguez e latino, Coimbra, 8 vols.

Cobarruvias, S. (1977): Tesoro de la lengua castellana o española, Madrid, Turner.

Cossío, J. M. (1926): "Don Juan de Iriarte y los jesuitas", BBMP, 227.

Cotarelo y Mori, E. (1897): Iriarte y su época, Madrid, Sucesores de Rivadeneyra.

Cotarelo y Mori, E. (1914): "La fundación de la Academia Española y su primer director

D. Juan Manuel F. Pacheco, Marqués de Villena", $B R A E$, I, 1, 4-38 y 2, 89-127.

Corominas, J. y Pascual, J. A. (1980-83): Diccionario crítico etimológico castellano e hispánico,. Madrid, Gredos, 5 vols.

Guigou y Costa, D. M. (1945): El Puerto de la Cruz y los Iriarte, Tenerife.

Iriarte, J. (1769): Regiae Bibliothecae Matritensis Codices Graeci Mss. Joannes Iriarte, ejusdem custos, manuscriptorum Museo olim praepositus, idemque Regis Interpres intimus, excussit, recensuit, notis, indicibus, anecdotis pluribus evulgatis illustravit. Opus Regis auspiciis et sumptibus in lucem editum. Volumen prius, Matriti, Typ. Antonii Perez de Soto.

Iriarte, J. (1771): Gramática latina escrita con nuevo método y nuevas observaciones. En verso castellano con su explicación en prosa, Madrid, Pedro Marín.

Lázaro Carreter, F. (1985): Las ideas lingüísticas en España durante el siglo XVIII, Barcelona, Crítica (1. ed. en Madrid, CSIC, 1949).

Menéndez Pelayo, M. (1974), Historia de las ideas estéticas en España, Madrid, CSIC, 2 vols.

Millares Carlo, A. (1981), Don Juan Iriarte, latinista y helenista, Las Palmas.

O'Scanlan, T. (1831): Diccionario marítimo español, Madrid, Imprenta Real (Facsímil en Madrid, Museo Naval, 1974).

Palau y Dulcet, A. (1954): Manual del librero hispanoamericano, Barcelona, Palau, vol. VII.

Real Academia Española (1726-1739): Diccionario de Autoridades, ed. facsímil, Madrid, Gredos, 1963, 3 vols.

Sempere y Guarinos, J. (1785-1789): Ensayo de una biblioteca de los mejores escritores del reynado de Carlos III, Nadrid, Imprenta Real, 6 vols., VI, pp. 181-190 (Facsímil en Madrid, Grados, 1969, 3 vols.).

Viera y Clavijo, J. (1777-1783), Noticias de la historia general de las Islas Canarias, Madrid, Blas Román, 4 vols., IV, 584 y ss.

Viñaza, Conde de la (1893): Biblioteca histórica de la Filología Castellana, Madrid, M. Tello, 3 vols. 